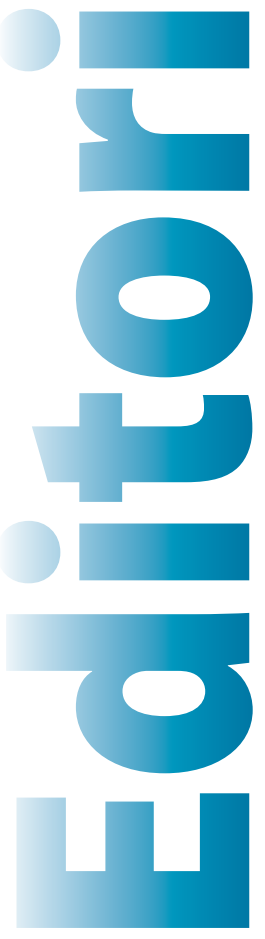

\title{
Nouvel arrêté plante... ça bouge... !
}

C'est peu dire que notre administration de tutelle, en l'occurrence la DGCCRF, se soit hâtée lentement de publier l'arrêté relatif aux plantes autorisées dans les compléments alimentaires. Depuis le décret de mars 2006 (transposition en droit français de la directive 2002, opposable depuis 2003... cherchez l'erreur !) qui régit les compléments alimentaires, voici enfin dirais-je, le texte tant attendu par l'ensemble des acteurs de la filière PMAI (Plantes médicinales, aromatiques et industrielles). $\mathrm{Vu}$ le nombre de compléments alimentaires à base de plantes

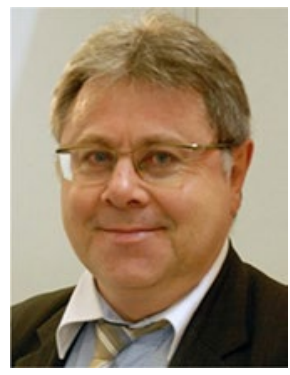
déclarés et mis sur le marché selon la procédure prévue à l'article 16 (Principe de reconnaissance mutuelle au niveau de la $\mathrm{CE}$ ), la situation était devenue ubuesque et non perdurable.

Après de multiples annonces de publications prochaines, malgré maint projets,

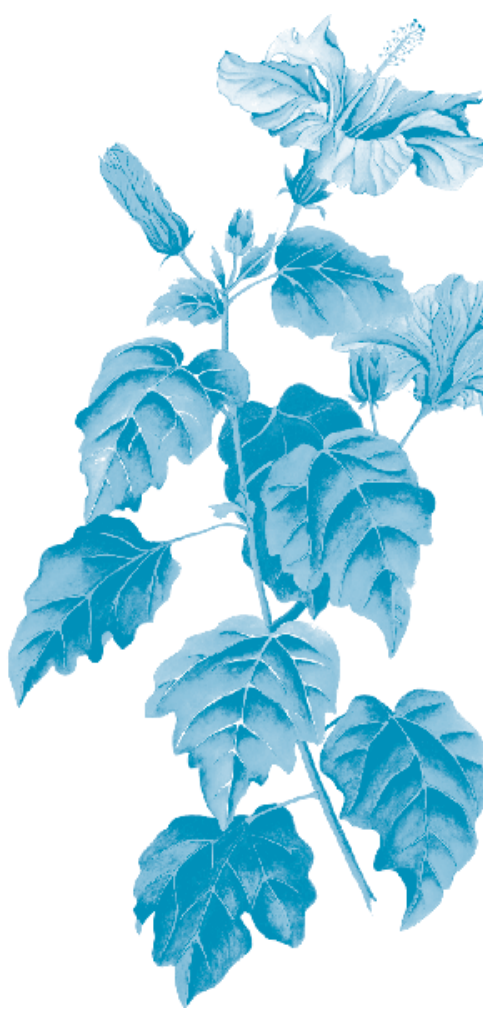
dont le dernier dénommé BelFrIt (Acronyme pour Belgique-France-Italie), tous ont été rejetés et/ou reportés dans le temps, donc renvoyés aux calendes grecques comme l'on dit en langage populaire. Puis tout à coup, comme une giboulée de mars imprévisible entre deux rayons de soleil, ballotée par les aléas des changements politiques, la publication de l'arrêté est tombée brutalement, comme une précipitation en plein milieu de ce mois de juillet 2014. Autrement dit 8 ans d'attente (voire davantage si l'on se réfère à la directive de 2002) pour un résultat qui laisse perplexe. En effet, le texte présente un manque de cohérence, des insuffisances et reflète apparemment un manque de concertation. Certes, à la décharge de la DGCCRF, il n'est pas aisé d'opérer entre le libéralisme et l'harmonisation européenne face au conservatisme et au protectionnisme des instances de santé.

Il est devenu commun de publier des textes importants quand la France se prélasse sur les plages. Vouloir faire passer le texte sous silence, c'est aussi prendre le risque qu'à retardement, il fasse grand bruit... Qu'à cela ne tienne! Avec les Simples (plantes médicinales unitaires) nous sommes depuis longtemps sensibilisés à la complexité. Nous ne sommes plus à un oxymore près pour oser qualifier ce texte d'une sombre clarté ! Ou l'inverse, tout dépend de quel côté on se place. Recto, cet arrêté présente l'avantage de constituer un cadre juridique permettant de clarifier la situation. Verso, il fait non seulement de l'ombre, mais assombri considérablement l'avenir de la phytothérapie en pharmacie d’officine, avec des répercussions inéluctables sur la pratique de la phytothérapie médicale. C'est dans la continuité du phénomène observé avec les teintures-mères. D’un côté, moins de 50 spécialités pharmaceutiques de médication familiales à base de plantes (consultation site ANSM juin 2014), de l'autre plusieurs dizaines de milliers de compléments alimentaires à base de plantes sur le marché français (tous circuits de distribution confondus). Le décret d'août 2008 avait déjà fait sortir 148 plantes du monopole pharmaceutique. C'est maintenant plus d'une 
centaine de drogues végétales inscrites à la pharmacopée et strictement médicinales qui se rajoutent et sortent du monopole. La brèche sétend à en devenir critique... Les pharmacognostes qui n’ont pas été concertés s'inquiètent, à juste titre... ne serions-nous pas une espèce en voie de disparition?

Dans ce numéro qui revêt ainsi un côté spécial, poussé par l'actualité, un article est consacré à cet arrêté. Il en détaille le contenu, émet l'hypothèse de son origine réglementaire pour mieux comprendre l'environnement instable de ce domaine et tente d'en prédire les conséquences. La présence de plantes nouvelles en alimentation (plantes de médecine traditionnelle chinoise, kampo, ayurvédique ou autres) soulève moult questions autour de la problématique des "produits dits frontières ", notamment celle centrale entre la dichotomie aliment/médicament versus le continuum entre effet nutritionnel, effets physiologique et médicinal...

En quelque sorte, cet arrêté renforce, de manière indirecte, le concept de nutraceutique au sens de produits de statut alimentaire qui présentent des bénéfices santé à finalité médicinale, bien au-delà du nutritionnel... Cette ambiguïté liée à la confusion entre l'idée que le complément alimentaire vise par définition à compléter le régime alimentaire normal de l'individu sain, et la réalité commerciale de vouloir exploiter leurs vertus médicinales dans des états physiopathologiques particuliers, car c'est la santé qui fait vendre, reflète un caractère d'amphibologie.

\section{Loïc Bureau}

Pr. associé en nutraceutique et responsable du master

"Ingénierie en nutraceutique",

faculté des sciences pharmaceutiques et biologiques, Université Rennes 1 et directeur d'IFAS (Institut de formation des acteurs de santé)

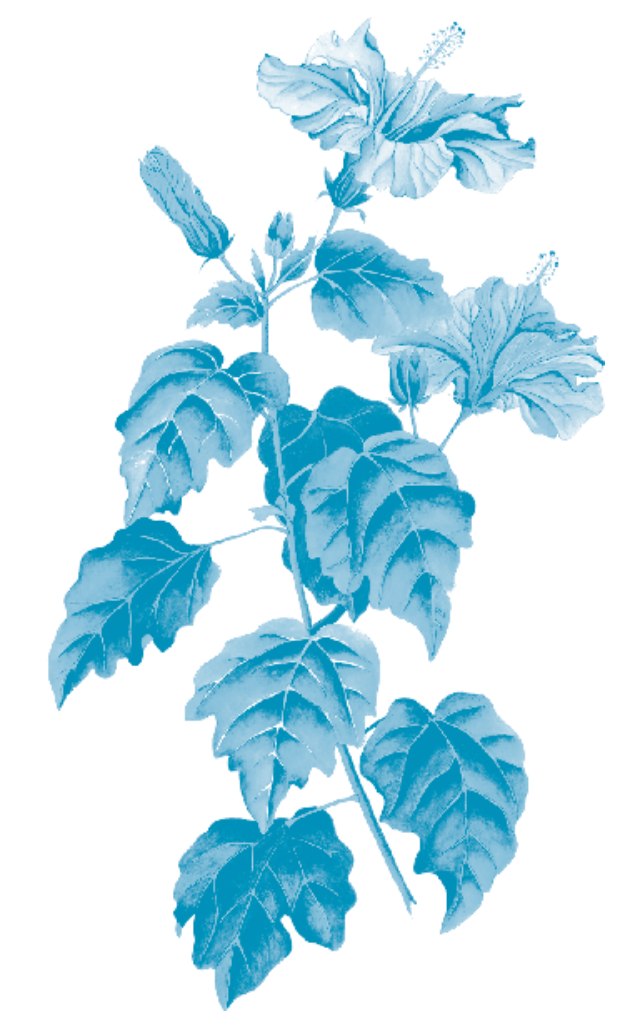

